\title{
La politique de gestion des eaux souterraines en France
}

Une analyse en termes de gestion intégrée

Groundwater Policy in France: an Integrated Management Analysis

\section{Olivier Petit}

\section{(2) OpenEdition}

\section{Journals}

Édition électronique

URL : http://journals.openedition.org/economierurale/300

DOI : $10.4000 /$ economierurale.300

ISSN : 2105-2581

Éditeur

Société Française d'Économie Rurale (SFER)

Édition imprimée

Date de publication : 15 février 2009

Pagination : 50-64

ISSN : 0013-0559

Référence électronique

Olivier Petit, «La politique de gestion des eaux souterraines en France », Économie rurale [En ligne], 309 | Janvier-février 2009, mis en ligne le 01 janvier 2011, consulté le 30 avril 2019. URL : http:// journals.openedition.org/economierurale/300; DOI : 10.4000/economierurale.300 


\section{La politique de gestion des eaux souterraines en France Une analyse en termes de gestion intégrée}

Olivier PETIT • Études et Recherches Économiques Interdisciplinaires de l'Artois (EREIA), Université d'Artois et Centre lillois d'études et de recherches sociologiques et économiques (Clersé), CNRS-Lille 1

a Gestion intégrée des ressources en eau L(GIRE) figure parmi les principes de gestion de l'eau défendus à l'échelle internationale depuis les années 1970. Entendue au départ comme une gestion par bassinversant, la notion a progressivement évolué pour désigner, dans une perspective inspirée de la systémique, une gestion de l'eau visant à prendre en compte les aspects qualitatifs et quantitatifs, le court terme et le long terme, les eaux de surface et les eaux souterraines, envisagée sous l'angle d'une gestion durable et concertée, organisée dans un cadre territorial cohérent - le bassin-versant pour les eaux de surface et le système aquifère pour les eaux souterraines - et reconnaissant l'eau comme un bien économique, dans le sillage des travaux engagés à la conférence de Dublin en 1992 (Petit, 2009). Bien que de nombreux débats persistent au plan international pour souligner le flou de cette notion (Biswas, 2004), elle nous paraît porteuse de transformations institutionnelles intéressantes à analyser et ouvre la voie à des recherches novatrices qui justifient que l'on mène une analyse empirique des mutations engendrées par sa prise en compte.

Depuis le milieu des années 1990, une évolution est perceptible en France en faveur d'une GIRE, en raison notamment d'une focalisation politique et médiatique sur la protection des eaux souterraines. Bien que les structures institutionnelles en charge de la gestion de l'eau en France soient longtemps demeurées cloisonnées, un effort de cohérence générale de la politique de l'eau - intégrant une variété d'usages et d'acteurs - conduit désormais à envisager celle- ci de manière plus transversale. Sous l'impulsion de la loi sur l'eau de 1992, mais aussi de la Directive cadre européenne sur l'eau (DCE) de 2000, plusieurs expériences attestent d'un changement dans la manière d'appréhender la ressource, et reconnaissent la nécessité de gérer l'eau tout au long de son cycle. Ce «décloisonnement »se traduit par exemple, dans le domaine de la police de l'eau, par la généralisation à l'échelle départementale, des Missions interservices de l'eau (MISE) qui permettent de mutualiser les compétences et de mener une politique beaucoup plus intégrée. Par ailleurs, depuis 1992, la mise en œuvre des Schémas directeurs d'aménagement et de gestion des eaux (SDAGE), qui conduisent les Agences à planifier la gestion de leur bassin dans un souci de cohérence de leurs actions, témoigne aussi de cette évolution.

S'agissant des eaux souterraines, si cellesci sont aujourd'hui reconnues comme un patrimoine qu'il est nécessaire de protéger et de gérer, la politique française de gestion de ces ressources n'est apparue que tardivement. Les outils de planification et de gestion des ressources en eau tels que les Schémas d'aménagement et de gestion des eaux (SAGE) et les contrats de rivière notamment ont d'abord été conçus pour gérer les eaux de surface, caractérisant ainsi une tendance à l'hydroschyzophrénie (Llamas, 1998), conduisant à penser la gestion des eaux souterraines indépendamment de celle des eaux de surface. Pourtant, au milieu des années 1990, une réflexion a été engagée par le Conseil général des Mines sur la transposition des 
SAGE aux eaux souterraines (Comte et al., 1995). La prise de conscience d'une nécessaire intégration des domaines et des politiques a conduit, sous l'impulsion de la DCE, à développer le suivi qualitatif et quantitatif des eaux souterraines, mais aussi à renforcer le rôle des instruments économiques, réglementaires et participatifs. En France, la Loi sur l'eau et les milieux aquatiques (LEMA) adoptée en $2006^{1}$ lève l'ambigüité des droits de propriété sur les eaux souterraines en leur attribuant un caractère domanial. À l'échelle européenne, l'adoption (en décembre 2006 également) de la directive « fille » sur les eaux souterraines renforce encore la politique de l'eau en ce domaine. Comment cette volonté de gestion intégrée se matérialise-t-elle dans la politique française de l'eau ? C'est ce que nous souhaitons analyser en prenant appui sur la politique française de gestion des eaux souterraines initiée depuis le début des années 1990. Pour cela, nous revenons d'abord sur la notion de la GIRE pour proposer un cadre de réflexion basé sur trois éléments : la dimension patrimoniale, les territoires de l'eau et les régimes institutionnels de ressources. Puis, nous analysons à partir de ces éléments la politique de l'eau en France dans la perspective d'une gestion intégrée.

\section{Jalons pour une analyse des politiques de gestion intégrée des ressources en eau}

La GIRE constitue un cadre de référence à l'échelle internationale et un objectif pour les politiques nationales de gestion de l'eau. Proposée à la conférence de Rio en 1992, réaffirmée à la conférence de Johannesburg en 2002, la mise en place de "Plans de gestion intégrée des ressources en eau et de gestion efficace de l'eau » dans tous les pays

1. Loi n ${ }^{\circ} 2006-1772$ du 30 décembre 2006 sur l'eau et les milieux aquatiques, Journal Officiel $n^{\circ} 303 \mathrm{du}$ 31 décembre 2006. était affichée comme un but à atteindre à l'horizon 2005, sans un réel consensus sur la signification précise de cette notion. Depuis quelques années, le Partenariat mondial de l'eau s'est fait le promoteur de la GIRE en proposant une définition qui fait aujourd'hui office de référence - «La gestion intégrée des ressources en eau désigne un processus qui favorise le développement et la gestion coordonnés de l'eau, des terres et des ressources connexes, en vue de maximiser, de manière équitable, le bien-être économique et social en résultant, sans pour autant compromettre la pérennité d'écosystèmes vitaux $»^{2}-$ malgré les ambigüités que certains auteurs ont dénoncé (Biswas, op. cit.). Par ailleurs, l'investissement fort d'institutions internationales en charge de sa promotion comme le Partenariat mondial de l'eau ou le Conseil mondial de l'eau, dont les liens avec les grands groupes privés sont assumés, rend cette notion sujette à caution. On peut y lire un moyen de promouvoir des politiques de l'eau basées sur des compromis difficiles à atteindre (reconnaissance de l'eau comme patrimoine commun et comme bien économique) qui tendraient, in fine, à soutenir un modèle économique libéral de désengagement des États, basé sur les partenariats public-privés, sous couvert de concertation ou de participation et de gestion par bassins (Petit, 2009). Sans vouloir minimiser ces critiques, il nous semble que cette notion n'a pas, jusqu'ici, fait suffisamment l'objet d'attention de la part de la communauté scientifique alors même qu'elle recèle un potentiel de problématiques et de changements institutionnels intéressants à analyser. En outre, le nombre limité de pays ayant adopté les plans préconisés et l'absence d'évaluation sérieuse de la mise en œuvre de ces plans doivent aussi inciter des travaux scientifiques sur le sujet (Petit, Baron, 2009). Pourtant, des

2. Global Water Partnership (2000). La gestion intégrée des ressources en eaux. TAC backgrounds papers, $\mathrm{n}^{\circ} 4,80 \mathrm{p}$. (voir en particulier la page 24). http://www.gwpforum.org/gwp/library/TAC4fr.pdf 
auteurs ont tenté, ces dernières années, d'apporter un éclairage théorique à cette notion. Calvo-Mendieta (2005) estime ainsi, en fondant son analyse sur quatre courants (les régimes institutionnels de ressources, l'économie de la proximité, l'économie des conventions et l'approche patrimoniale), que ceux-ci permettent de mieux circonscrire l'ensemble des problématiques couvertes par la GIRE, en lien avec la thématique des conflits d'usage. L'auteure propose une définition de la GIRE $^{3}$ fondée sur ces approches, prenant en compte trois dimensions ${ }^{4}$ :

- «la prise en considération des variables spatiales;

- [...] l'importance des institutions (formelles et informelles);

- [...] le rôle des formes de régulation publique dans la gestion des conflits »

Dans le même esprit, nous souhaitons avancer trois axes d'analyse pour évaluer les politiques de l'eau au regard de cette notion. Ils font écho aux dimensions relevées cidessus, mais s'en distinguent néanmoins. Un processus de la GIRE doit s'appuyer, selon nous, sur :

- la prise en compte de la dimension patrimoniale de l'eau ;

- la reconnaissance des territoires de l'eau ; - un régime institutionnel de ressources intégré.

Ces trois éléments ne couvrent pas tous les aspects de la gestion intégrée, mais visent à poser les jalons d'une analyse des processus de gestion de l'eau, soulignant les caractères juridique, institutionnel, économique et territorial de tout processus de gestion intégrée.

3. Selon Calvo-Mendieta (2005), «la définition d'une gestion intégrée des ressources en eau nécessite : 1. un régime institutionnel de ressources en eau intégré (forte cohérence entre politiques publiques et droits de propriétélusage et forte étendue) ; 2. la reconnaissance et compréhension des conflits d'usage et de leur dépassement ; 3. la transversalité des politiques publiques ayant une influence sur l'eau, notamment les politiques de gestion de l'espace. » (In page 24).

4. In Calvo-Mendieta, 2005, page 316.

\section{La dimension patrimoniale}

Le premier niveau d'analyse permettant d'évaluer la progression des politiques publiques vers une gestion intégrée concerne la dimension patrimoniale que l'on retrouve aujourd'hui dans nombre de textes réglementaires et légaux encadrant la gestion de l'eau à travers le monde. Cette dimension est présente dans la loi française sur l'eau de 1992 qui reconnaît que «l'eau fait partie du patrimoine commun de la nation » (art. 1). Elle est également assumée, quoique dans une formulation plus ambigüe, dans le préambule de la DCE de 2000 qui dispose que «l'eau n'est pas un bien marchand comme les autres mais un patrimoine qu'il faut protéger, défendre et traiter comme tel».

D'un point de vue théorique, on assiste depuis quelques années à un renouveau des analyses en sciences sociales en termes de patrimoine, et leurs applications aux ressources en eau nous paraissent fécondes. Une première vague de travaux, initiés au milieu des années 1970 dans le cadre de recherche-actions pilotées par le bureau de Rationalisation des choix budgétaires - RCB - du ministère français de l'Agriculture, a déjà pris les eaux souterraines pour champ d'investigation (Ollagnon, 1979). Ces travaux pointaient la nécessité d'institutions aptes à prendre en charge la gestion patrimoniale de la qualité. Ils ont donné lieu, au milieu des années 1980, à un ouvrage de synthèse (Montgolfier et Natali, 1987) mettant en évidence une série de méthodes visant à dépasser les analyses coût-bénéfice dans le domaine de la gestion des ressources naturelles et de l'environnement. L'audit patrimonial (Ollagnon, 1987) et les démarches de médiation patrimoniale appliquées dans les pays en développement (Babin et al., 2002) s'inspirent ou prolongent cette approche mais font l'objet de critiques touchant notamment à la méthodologie adoptée et à ses résultats (Mermet, 2007).

Plus récemment, un groupe d'économistes (Barrère et al., 2005) a entrepris un travail conceptuel de définition d'une 
économie du patrimoine, se dissociant des travaux de gestion et d'audit patrimonial, sans renier pour autant tous les apports conceptuels de ces démarches. Ces auteurs revendiquent la dimension patrimoniale de tout acte économique qui doit être analysée de manière décloisonnée des modes d'allocation et de gestion marchande. Ils s'appuient sur des travaux de l'ancienne économie institutionnelle, de sociologie et d'anthropologie économique, permettant d'aller au-delà de la vision purement individualiste du patrimoine. Pour ces auteurs, les «économistes du marché » se sont approprié la catégorie analytique du patrimoine pour tenter de rabattre le nonmarchand vers du marchand en mobilisant les notions d'externalité, de bien public, de capital social ou naturel par exemple. Cette requalification du non-marchand en marchand autorise alors l'usage de méthodes d'évaluation monétaire qui dénaturent le patrimoine en lui prêtant des attributs qui peuvent lui être étrangers. L'économie du patrimoine propose un cadre théorique permettant de penser à la fois les relations marchandes et non marchandes, en analysant la coexistence de la propriété privée et du patrimoine commun. Il devient dès lors nécessaire de reconnaître une diversité de rationalités économiques « et en particulier, celle qui vise à maintenir l'identité des communautés humaines en tant qu'elles s'inscrivent dans le temps long du renouvellement des générations et des dynamiques des espaces naturels » (Barthélemy et al., $2005)^{5}$. Par ailleurs, ces auteurs postulent l'existence d'une relation identitaire fondée sur un rapport spécifique au temps et à l'espace. D'un point de vue méthodologique, l'économie du patrimoine appelle au «désencastrement » des relations marchandes et souligne la nécessité de mettre en relation le rapport marchand « avec un autre rapport économique de

5. In Barthélemy et al., 2005, page 131. même niveau sémantique [...] d'essence patrimoniale » (Barthélemy et al., 2005). Notons que cette représentation idéaltypique de l'économie du patrimoine n'est pas plus réelle que la formalisation d'une économie marchande en situation de concurrence pure et parfaite. Toutefois, elle permet de penser les relations entre acteurs comme étant issues de l'influence de relations marchandes et patrimoniales.

Cette approche est intéressante pour notre analyse des processus de la GIRE. Elle insiste sur l'émergence d'institutions patrimoniales résultant de l'influence des relations marchandes et non marchandes dans la société. Par ailleurs, elle permet de penser la coexistence du patrimoine commun et de la propriété privée et de montrer que certains biens, comme l'eau, sont dans une tension perpétuelle entre ces deux bornes. Les institutions qui tentent de concilier les usages concurrents, de trouver des figures de compromis intégrant à la fois la dimension marchande de l'eau et sa dimension patrimoniale seraient dès lors, des marqueurs d'une gestion intégrée.

\section{La reconnaissance des territoires de l'eau}

Plusieurs travaux s'attachant à la gestion spatiale (Narcy, 2004) ou territoriale (Ghiotti, 2007) de l'eau peuvent également être mobilisés pour tenter de construire un cadre d'analyse de la mise en œuvre de la GIRE. Ils tentent de trouver des outils pour s'interroger sur la persistance de cloisonnements dans le domaine de la gestion de l'eau et dans ses rapports aux politiques d'aménagement du territoire. La pertinence du bassin-versant comme unité territoriale et politique cohérente pour la gestion de l'eau est alors analysée. Narcy (op. cit.) met ainsi en évidence les disjonctions entre politiques de gestion de l'eau et de gestion des espaces. Il souligne la nécessité d'un dépassement de la gestion «fluxiale » de l'eau pour aller vers

6. Cf. Barthélemy et al., page 139. 
Tableau 1. Typologie des régimes institutionnels de ressources (RIR)

\begin{tabular}{l|l|c|c}
\hline \multirow{2}{*}{ Types de RIR } & \multicolumn{2}{c}{ Cohérence } \\
\cline { 3 - 4 } Étendue & Faible & RIR inexistant & Élevée \\
\cline { 2 - 4 } & Élevée & RIR complexe & RIR intégré \\
\hline
\end{tabular}

une gestion spatiale. Cette transition indique l'importance d'une prise en compte d'une rationalité politique à côté de la rationalité technique. En insistant sur la nécessité d'un passage d'une vision techniciste de l'homme maître et possesseur de la nature à une vision plus politique de l'homme dans la nature, l'auteur pointe les limites des modes de gestion cloisonnés et appelle à la reconnaissance, dans les processus de gestion, des facteurs de blocage concourant, selon nous, à une dynamique de gestion intégrée de l'eau et des territoires. Ghiotti (op. cit.) se focalise, pour sa part, sur la rencontre du monde de la gestion de l'eau et sa logique de bassin-versant et celui des territoires qui se sont construits indépendamment de toute référence au bassin. S'appuyant sur plusieurs exemples de SAGE et de contrats de rivières dans les montagnes méditerranéennes françaises, il montre que la logique de bassinversant, souvent présentée comme « évidente », bute sur un certain nombre de contradictions qui tiennent notamment au poids prépondérant des autres découpages territoriaux (départements, régions, intercommunalités) : « [L]es liens tissés entre eau et développement montrent que, en devenant un territoire politique, le bassin-versant se trouve rattrapé par une logique qu'il devait dépasser pour constituer une forme hybride au carrefour des influences politiques, administratives, socio-économiques et environnementales. Le territoire problème devient territoire porteur de stratégies et de potentialités. Mais les potentialités et les ressources ne sont pas les mêmes selon les collectivités et les transferts de compétences prévus pourraient aggraver les dispa-

7. Cf. Ghiotti, 2007, p. 217. rités et les inégalités territoriales $\gg^{7}$. Dès lors, l'un des objectifs de la gestion intégrée est de s'appuyer sur la diversité des territoires de l'eau ${ }^{8}$, dans leurs dimensions à la fois naturelle et politique.

\section{Les régimes institutionnels de ressources}

Les travaux sur les régimes institutionnels de ressources (RIR) apportent, d'un point de vue théorique, des fondements à la GIRE, dépassant une vision qui en ferait l'objet de consensus entre les acteurs. Ces travaux soutiennent que si l'objectif de la gestion intégrée est de dépasser les conflits d'usage, il faut reconnaître au conflit son rôle structurant dans les rapports humains. La gestion intégrée permet alors de s'inscrire dans un processus de recherche d'un compromis institutionnalisé (Sangaré et Larrue, $2002^{9}$; Calvo-Mendieta, op. cit.).

Les RIR permettent d'étudier de manière dynamique les arrangements institutionnels qui se forment, en tenant compte des droits de propriété sur les ressources naturelles et des politiques publiques qui régulent la protection et l'exploitation de ces ressources (Varone et al., 2008) ${ }^{10}$. Signalons que les RIR sont caractérisés par leur étendue et

8. L'eau ne constitue d'ailleurs qu'un cas particulier des multiples formes prises par les espaces de régulation des problèmes environnementaux. Salles (2006) a bien montré cette diversité des territoires d'environnement.

9. I. Sangaré, C. Larrue (2002). The Evolution of the National Water Regime in France. Euwareness report, April, http://www.euwareness.nl

10. F. Varone, S. Nahrath, J.-D. Gerber (2008). Régimes institutionnels de ressources et théorie de la régulation. Revue de la régulation, $\mathrm{n}^{\circ} 2$, Varia, mis en ligne le 31 janvier 2008, http://regulation. revues.org 
leur cohérence : "L'étendue d'un RIR fait référence au nombre de biens et services régulés par le régime à un temps donné. Le critère de cohérence renvoie au contenu et à l'articulation entre elles des différentes régulations de droit public [...] et privé [...] constitutives du régime» (Varone et al., $o p$. cit. $)^{11}$. Ces caractéristiques permettent finalement de distinguer quatre types de RIR (tableau 1).

Ce cadre analytique a notamment été appliqué aux régimes institutionnels de l'eau dans le cadre d'un projet de recherches européen qui s'est déroulé entre 2000 et 2002, EUWARENESS, et qui a permis de confronter l'évolution des RIR dans différents pays européens.

Finalement, dans la mesure où la gestion intégrée est toujours une tentative de réconciliation d'intérêts ou de droits différents, voire contradictoires, de conflits d'usage, de territoires aux multiples légitimités, il nous paraît intéressant de regarder de plus près la manière dont les eaux souterraines ont été progressivement intégrées dans les politiques publiques de gestion de l'eau en France, pour y lire ces tensions comme le témoignage d'un pas franchi vers une gestion intégrée.

\section{Politique de gestion de l'eau et intégration progressive des eaux souterraines}

L'analyse et l'évaluation des politiques publiques peuvent recouvrir diverses formes, suivant les objectifs qui leur sont assignées. Ainsi, notre analyse de la politique de gestion des eaux souterraines menée en France depuis le début des années 1990 n'a pas pour ambition de mesurer sa performance à partir d'une batterie d'indicateurs, ni d'engager un travail de réflexion collective avec les acteurs de ces politiques en vue de leur amélioration. Notre but est

11. In Varone et al., voir en particulier pages 9-10. plutôt celui d'une production de connaissances permettant de mieux comprendre les enjeux de renouvellement de l'action publique par rapport à un objectif posé, à l'échelle internationale, comme devant guider les politiques de gestion de l'eau. Cette analyse est basée sur trois éléments présentés dans la première section : la reconnaissance de la dimension patrimoniale, la prise en compte des territoires de l'eau et la qualification du régime institutionnel de l'eau. Il convient néanmoins, avant de mener cette analyse, de présenter brièvement l'état qualitatif et quantitatif des eaux souterraines en France.

\section{Le suivi qualitatif et quantitatif des eaux souterraines}

Jusqu'à la fin des années 1990, il n'existait pas de mise en cohérence globale des informations relatives aux eaux souterraines. Martin (1996) relevait dans son rapport sur la gestion durable des eaux souterraines le manque de connaissances exhaustives tant de la qualité que de la quantité d'eau présente et prélevée. Parmi les mesures qu'il préconisait, figurait la mise en place d'une banque de données afin d'assurer ce suivi. Cette proposition a eu, semble-t-il, un écho auprès de la direction de l'Eau du ministère en charge de l'Environnement puisqu'au début de l'année 2003 a été lancée l'Accès aux données sur les eaux souterraines (ADES) dont la maittrise d'œuvre a été confiée au Bureau de recherches géologiques et minières (BRGM). Les données contenues dans la base fournissent un outil de communication et de mise à disposition des informations sur les eaux souterraines permettant de répondre aux exigences de la DCE. Ce dernier texte exigeait en effet que des réseaux de surveillance qualitative et de suivi piézométrique des eaux souterraines soient mis en place pour la fin de l'année 2006.

Rappelons qu'il existe en France environ 6500 aquifères dont $20 \%$ (1300 environ) possèdent une superficie de $10 \mathrm{~km}^{2}$ au moins. Deux cents d'entre eux (175 nappes 
libres et 25 nappes captives) atteignent une dimension régionale ou plus (de 100 à $100000 \mathrm{~km}^{2}$ ) et offrent des ressources exploitables. Ces 200 aquifères totalisent un volume de 2000 milliards de $\mathrm{m}^{3}$ d'eau potentiellement exploitables et 100 milliards de $\mathrm{m}^{3}$ s'écoulent annuellement de ces aquifères vers la mer (en majorité) et les cours d'eau exutoires, « représentant la plus grosse partie du débit de ces cours d'eau » (BRGM, 2006) $)^{12}$. En flux annuel moyen, la France métropolitaine reçoit un volume de 182 milliards de $\mathrm{m}^{3}$ de pluies efficaces, dont plus de la moitié (environ 100 milliards) sert à alimenter les eaux souterraines (ce chiffre tenant compte des transferts avec les pays frontaliers). Au niveau des prélèvements, on estime qu'environ 6,3 milliards de $\mathrm{m}^{3}$ sont prélevés annuellement dans les eaux souterraines pour l'ensemble des besoins humains ${ }^{13}$. Parmi les usages de l'eau, c'est essentiellement l'Alimentation en eau potable (AEP) qui sollicite ces ressources (59\% des prélèvements), suivie des prélèvements pour l'industrie $(23 \%)$ et pour l'agriculture (18\%) (IFEN, 2004). Mais si le montant global des prélèvements annuels dans les eaux souterraines est largement inférieur à la recharge, ces chiffres ne doivent pas masquer de grandes disparités et l'existence de phénomènes localisés de surexploitation (lorsque les prélèvements sont supérieurs à la recharge).

D'un point de vue qualitatif, la présence de nitrates et de pesticides est souvent un marqueur de qualité, même si de nombreux autres paramètres sont suivis. À ce sujet, une étude menée en 2002 et compilée par le $\mathrm{RNES}^{14}$ montre que $62 \%$ des 1048 sites

12. BRGM (2006). Les eaux souterraines en France en quelques chiffres. BRGM, Paris, note du 18 mai 2006, voir en particulier la page 1 . http://www.brgm.fr

13. Le montant global des prélèvements annuels (eaux souterraines et eaux de surface) s'élève à 34 milliards de $\mathrm{m}^{3}$.

14. Réseau national de connaissance des eaux souterraines. échantillonnés présentent une concentration de nitrates supérieure à $10 \mathrm{mg} / \mathrm{l} ; 10 \%$ des points de mesure révèlent une concentration comprise entre 50 et $100 \mathrm{mg} / \mathrm{l}$ et $1 \%$ seulement une concentration supérieure à $100 \mathrm{mg} / \mathrm{l}$. Mais, comme le souligne l'IFEN, le système de mesure n'est pas nécessairement représentatif et «indiquer que pour $62 \%$ des stations les teneurs en nitrates sont telles qu'elles traduisent une influence anthropique ne signifie pas que $62 \%$ des nappes sont contaminées $\gg^{15}$. Prises en dynamique pourtant, les données compilées montrent une nette tendance à la dégradation de la qualité de l'eau, dans la mesure où $32 \%$ des stations montrent une élévation significative de la présence de nitrates entre 1992 et 2000-2001 (IFEN, op. cit.). La même étude du RNES a permis de quantifier la présence de pesticides (insecticides, herbicides et fongicides) dans $60 \%$ des 1078 points de surveillance. L'IFEN (2004) précise que «sur l'ensemble des points de mesure quantifiés, $41 \%$ sont altérés à un niveau tel que, si ces ressources étaient utilisées pour la production d'eau potable, elles nécessiteraient un traitement préalable spécifique aux pesticides ${ }^{16}$. Soulignons que la répartition spatiale des eaux altérées montre en moyenne des concentrations beaucoup plus importantes dans le nord de la France qu'au centre et dans le sud. Néanmoins, quelques points de mesure présents dans le bassin Rhône Méditerranée Corse signalent une eau inapte à la production d'eau potable.

Ainsi, la lente dégradation de la qualité des eaux souterraines (en particulier dans le quart nord-ouest du territoire), souligne le coût croissant de dépollution que les ménages doivent prendre en charge pour l'AEP, mais aussi certaines limites de la politique française de l'eau, incapable jusqu'ici d'inverser cette tendance. Par ailleurs, signalons que l'interdépendance

15. IFEN, 2004, p. 18.

16. IFEN, 2004, p. 22. 
des aspects qualitatifs et quantitatifs est cruciale pour les eaux souterraines car la surexploitation de ces ressources peut, par exemple, engendrer des problèmes qualitatifs non négligeables (Petit, 2004).

\section{La reconnaissance de la dimension patrimoniale : évolution du régime juridique et des politiques publiques}

Le régime juridique des eaux de surface a connu une évolution notable à partir du $\mathrm{XIX}^{\mathrm{e}}$ siècle. C'est à cette époque en effet que le régime juridique des cours d'eau passe « d'un système où se lisait la concurrence entre trois pôles de souveraineté - les pouvoirs central, seigneurial et communautaire - à une configuration où se révèle cette fois ce que B. Barraqué (1997) désigne par un "système en tension État/propriétaire”, opposant deux types de souveraineté : celle conférée au citoyen par la propriété privée, et celle de l'État jacobin. » (Narcy, op. cit. $)^{17}$. Le régime juridique des eaux souterraines est demeuré en revanche, jusque très récemment, dans une position très incertaine. Billet écrivait ainsi en 2001 :

«Le mutisme du législateur a divisé et divise toujours la doctrine. Les eaux souterraines sont considérées par certains comme des res nullius, des choses sans maitre qui ne peuvent être appropriées que par occupation. [...] D'autres auteurs estiment, au contraire, que l'eau qui se trouve enfermée dans le sol est une partie intégrante du fonds [...] et qu'elle appartient en conséquence au propriétaire du sol en vertu de l'article $552 \mathrm{du}$ Code civil. D'autres enfin, laissent prudemment la question en suspens. Ces divergences doctrinales peuvent sans doute s'expliquer par les incertitudes de la jurisprudence, qui a longtemps hésité entre l'appropriation par accession et l'appropriation par occupation des eaux souterraines, pour finalement opter pour la dernière solution. [...] Face à ces incertitudes, ne devrait-on cependant pas opter pour une qualification plus conforme à la réalité et considérer les eaux souterraines comme des res communes au sens de l'article $714 \mathrm{du}$ Code civil, choses qui n'appartiennent à personne mais dont l'usage est commun à tous ? » (Billet, 2001, pp. 405-406).

17. In Narcy, page 80.
Malgré la loi sur l'eau de 1992, qui établit une réglementation sur les prélèvements dans les eaux souterraines, le caractère ambigu de la propriété de ces eaux demeure. Barraqué (1999) relève à leur propos que «[s] les eaux de surface sont de plus en plus considérées comme "publiques", les eaux souterraines sont ignorées car vécues comme "privées". D'où certains excès dans l'exploitation des nappes souterraines peu profondes, parce que non soumises, comme les eaux de surface, à des règles d'usage bien acceptées et donc faciles à appliquer ». La loi de 1992 reconnaît l'unicité de la ressource et met en évidence la nécessité d'une gestion globale et équilibrée. Elle insiste également sur la reconnaissance de l'eau comme « patrimoine commun de la nation » (art. 1), ce qui renforce un peu plus l'ambigüité relevée par Billet (2001).

À l'échelle européenne, la DCE du 23 octobre 2000 va enclencher un processus favorable à la connaissance et à la protection des ressources en eau en général et à la protection des eaux souterraines notamment. L'objectif général de cette directive, qui vise à atteindre un bon état qualitatif et quantitatif des masses d'eau en Europe à l'horizon 2015, a poussé à l'adoption de mesures de gestion conjointe des eaux de surface et des eaux souterraines et participe donc du mouvement de la GIRE. Cette directive souligne en particulier les interdépendances entre eaux de surface et eaux souterraines (préambule, al. 33), entre problèmes qualitatifs et quantitatifs (voir al. 34, en particulier) et reconnaît que "l'état quantitatif d'une masse d'eau souterraine peut avoir une incidence sur la qualité écologique des eaux de surface et des écosystèmes terrestres associés à cette masse d'eau souterraine » (préambule, al. 20). Parmi les objectifs assignés à cette directive, figure notamment celui de bon état des eaux souterraines. Annoncée depuis plusieurs années, une directive « fille » 
sur les eaux souterraines ${ }^{18}$ a été adoptée le 12 décembre 2006 et renforce encore les objectifs de protection des eaux souterraines en Europe en définissant des étapes pour la caractérisation du bon état chimique des masses d'eau souterraine. Tout comme la DCE, elle dresse un calendrier pour inciter les États membres à définir, d'ici le 22 décembre 2008, une valeur seuil pour chaque polluant identifié dans leurs eaux souterraines jugées à risque. Elle vise également à définir, dans chaque district hydrographique, des mesures de prévention et de limitation des rejets indirects dans les eaux souterraines. Elle indique également qu' afin de limiter les rejets polluants dans les eaux souterraines, les États se partageant des masses d'eau souterraine doivent se coordonner pour mener à bien le travail d'identification des substances à risque et de détermination des seuils (art. 16). Par ailleurs, la directive fille souligne, dans le même esprit que la DCE, qu'en complément des mesures préconisées dans le texte, les États peuvent mobiliser les instruments législatifs, administratifs et négociés pour accompagner leur programme de mesures.

La LEMA, adoptée en décembre 2006, tente d'apporter une solution à l'ambigüité du statut juridique des eaux souterraines en faisant désormais dépendre les eaux souterraines du régime de domanialité. Cette modification importante permet de rapprocher les eaux souterraines du régime qui opère sur les eaux de surface. Sangaré et Larrue (2002) ${ }^{19}$ notent la relative stabilité du régime juridique de l'eau en France depuis la loi du 8 avril 1898. Celui-ci se caractérise par un développement de la propriété privée, restreint par certaines dispositions limitant les usages afin de rendre l'eau accessible au plus grand nombre. La prise en compte de la dimen-

18. Directive 2006/118/CE.

19. Dans le présent article, voir la note 9. sion patrimoniale, avec la loi sur l'eau de 1992 et la DCE de 2000 vient toutefois renforcer le caractère collectif des structures institutionnelles en charge de l'eau. D'une certaine manière, le régime juridique des eaux souterraines, en se situant à l'interface entre propriété privée, régime domanial et patrimoine commun, témoigne de la volonté de protéger ces ressources pour l'ensemble des usages, y compris comme support de vie des écosystèmes. La dimension patrimoniale se retrouve intégrée aussi au niveau des politiques publiques à travers la mise en place de SAGE (pouvant être considérés comme des institutions patrimoniales) qui permettent de définir un périmètre et une communauté de cogestionnaires (les membres de la Commission locale de l'eau) renforçant la dimension identitaire attachée au territoire sur lequel cette gestion s'exerce. Les comportements individuels demeurent toutefois importants (persistance de la propriété privée), mais ceux-ci sont régulés grâce à des procédures formelles ou informelles faisant appel aux instruments réglementaires (interdictions, autorisations, zones de répartition des eaux), économiques (application des redevances notamment) et participatifs. La LEMA, en affirmant le caractère domanial des eaux souterraines, devrait donner plus de poids aux préfets pour appliquer des mesures réglementaires sur ces ressources. L'intervention des préfets était en effet conditionnée jusqu'alors par des situations « exceptionnelles » qui nécessitaient que des mesures, elles aussi exceptionnelles, soient prises. Dès lors que le caractère domanial des eaux souterraines est reconnu, les préfets ont toute légitimité pour prendre des arrêtés.

La coexistence de la propriété privée et du patrimoine permet donc, comme Barthélemy et al. (2005) le suggèrent, de comprendre certaines des mutations qui se sont opérées dans les politiques publiques dans le domaine de l'eau en France. 


\section{Une prise en compte progressive des territoires de l'eau}

La prise en compte des territoires de l'eau se manifeste par la transition qui s'opère, au niveau des politiques de l'eau, dans la reconnaissance des interdépendances entre eau de surface et eau souterraine. Si les procédures de contractualisation dans le domaine de l'eau (contrats de rivière) et les SAGE étaient prioritairement dédiés, jusqu'au milieu des années 1990, aux eaux de surface, la création de plusieurs contrats de nappe et de SAGE spécifiquement dédiés aux eaux souterraines, puis l'intégration dans ces processus institutionnels des interdépendances nappes-rivières, témoignent d'une évolution allant dans le sens d'une gestion intégrée. La loi sur l'eau de 1992 préconise la création de SAGE en tant qu'outil de coordination des ressources en eau au niveau de sous-bassins. Or, pour les eaux souterraines, l'unité d'analyse «bassin-versant » n'est pas proprement adéquat. L'équivalent du bassin-versant pour l'eau souterraine est le système aquifère, c'est-à-dire "l'ensemble d'un réservoir naturel souterrain et de l'eau qu'il contient ou qui le traverse $»^{20}$.

Les premiers SAGE mis en place concernaient plutôt de petits bassins hydrographiques mais la nécessité d'étendre cette procédure aux aquifères n'est apparue qu'au milieu des années 1990. Une étude commandée par la direction de l'Eau du ministère de l'Environnement et conduite en 1995 par le Conseil général des Mines (Comte et al., 1995) prend acte de cette nécessité, même si d'autres propositions, concernant notamment la notion de contrat de nappe sont envisagées. Ces formes de contractualisation sont particulièrement intéressantes à mentionner car, comme le rappelle Ghiotti (op. cit.) $)^{21}$ qui s'appuie sur les travaux que Salles et Zelem (1997) ont consacré aux contrats de rivière, «[1]es politiques contractuelles attestent un double

20. Cf. J.P. Comte et al. (1995, p. 3).

21. In Ghiotti, page 16. processus de transition territoriale et politique ». Le premier contrat de nappe signé portait sur la nappe d'Alsace (1990-1993), l'une des plus grandes réserves d'eau souterraines en Europe. Les contrats de nappe successifs ont permis de financer une dizaine de projets chaque année. Afin de servir de centre de ressources et de suivi des réseaux d'observation de la nappe d'Alsace, une association a vu le jour en 1995. Elle mène des opérations de sensibilisation, permet la mise à disposition d'informations sur la nappe d'Alsace auprès des acteurs qui en font la demande et exploite un modèle hydrodynamique régional. Les contrats de nappe ont chacun leurs particularités institutionnelles. Si l'initiative est souvent régionale, la mise en œuvre et le suivi dépendent de la configuration institutionnelle choisie par les acteurs en présence. Par exemple, le suivi du contrat de nappe des sables astiens est effectué par l'intermédiaire d'un «Comité de nappe », par analogie avec les «Comités de rivières » des contrats de rivières. L'une des limites de ces contrats de nappe tient au caractère volontaire de la démarche. Bien que ces contrats soient partie intégrante des SDAGE dans les différents bassins hydrographiques qui se sont dotés de cet outil, l'animation du contrat et le respect des engagements est souvent difficile à trouver, faute de mesures réglementaires. C'est pour cette raison que certains contrats de nappe donnent lieu, à l'issue du contrat, au lancement d'une procédure SAGE afin d'institutionnaliser la démarche et de permettre de donner un statut réglementaire aux décisions prises par le comité de nappe.

L'application des SAGE aux eaux souterraines s'est concrétisée au milieu des années 1990. Martin (1996), en conclusion d'un rapport sur la gestion durable des eaux souterraines, n'était guère enthousiaste : «Parce qu'il n'existe aujourd'hui, pratiquement rien dans le domaine de la gestion collective des aquifères et parce que le problème lui-même des liaisons entre eau souterraine et cours d'eau n'est pas (ou est 
mal) posé, il faut donner la priorité à la constitution de SAGE dont la zone de compétence soit celle d'un aquifère important (nappe de Beauce, aquifère de Gironde, Bassin ferrifère lorrain, nappe d'Alsace, grands karsts) ou modeste (ASTIEN). Il est peu probable que les élus poussent à la constitution de SAGE conçus pour gérer des aquifères importants; il serait bon que l'administration prenne l'initiative de les proposer $»^{22}$. Plusieurs SAGE intègrent aujourd'hui les interdépendances entre eau de surface et eau souterraine, y compris ceux qui, dans un premier temps, avaient été conçus spécifiquement pour gérer les eaux souterraines comme le SAGE « nappe de Beauce » (Petit, 2006).

Ces deux outils (SAGE et contrats de nappe) ne permettent pas cependant de couvrir toute la gamme des politiques publiques dédiées, directement ou indirectement, à la gestion des eaux souterraines. En effet, de nombreuses politiques sectorielles, à commencer par les politiques agricoles, se positionnent, sur un territoire généralement différent du bassin-versant ou du système aquifère, pour proposer des solutions aux problèmes de pollution ou de gestion quantitative des eaux de surface et des eaux souterraines. Mentionnons à titre d'exemple toute la gamme des instruments lancés dans les années 1990 par l'Association nationale pour le développement agricole (ANDA) (programmes Irri-Mieux, Ferti-Mieux, Phyto-Mieux). Les instruments contractuels poussent à la «normalisation des usages agricoles au nom de la protection d'un bien commun » (Busca et al., 2003) ${ }^{23}$. Plus précisément, «[c]es dispositifs se distinguent essentiellement par leur degré d'intégration (du sectoriel au multisectoriel) et prennent la forme soit d'actes publics de type contractuel ou de dispositions incitatives (conventions, chartes, partenariats qui renvoient ici plus au processus de

22. Martin Y., 1996, p. 34.

23. In Busca et al., page 309. construction d'accords entre les différents partenaires qu'à l'acte juridique qui les unit), soit d'actes réglementaires a priori plus coercitifs » (Ibid.). Ces initiatives locales et sectorielles, couplées aux instruments plus spécifiquement dédiés à la gestion de l'eau, participent d'une reconfiguration de l'action publique territoriale, allant vers davantage d'intégration. Tous ces processus visant à l'intégration des politiques ne sont cependant pas nécessairement des succès car, en déplaçant les frontières de l'action publique, on multiplie parfois aussi les problèmes et les conflits. Toutefois, la lenteur de certaines procédures très intégrées d'emblée (SAGE par exemple) peut trouver des relais dans des politiques contractuelles beaucoup plus ciblées (programmes Irri-Mieux, par exemple) qui participent d'un mouvement qu'il convient d'analyser de façon conjointe.

Cette transition vers une reconnaissance des territoires de l'eau est également le fruit de plusieurs dispositions légales et réglementaires. La loi sur l'eau de 1992 reconnaît ainsi l'unicité de la ressource et la nécessité d'une gestion globale. De même, la DCE, en posant le principe d'une gestion qualitative et quantitative des masses d'eau, renforce cette tendance mais le décloisonnement persistant entre gestion de l'eau et filières de gestion des espaces relevé par Narcy ( $o p$. cit.) témoigne des difficultés rencontrées pour mener véritablement une politique intégrée en ce domaine. Si l'adoption de la LEMA confirme une transition (amorcée dans les années 1980 et affirmée dès la loi sur l'eau de 1992), qui permet de penser à la fois la gestion de l'eau et des milieux aquatiques, prenant acte des interdépendances créées par l'eau, d'autres domaines d'intervention montrent que l'eau n'est pas véritablement intégrée aux politiques mais constitue encore un élément de contrainte. À cet égard, malgré les mutations de l'action publique locale dans le domaine de l'eau engendrées par les démarches contractuelles et la création d'un grand ministère de l'Éco- 
logie, du Développement et de l'Aménagement durable, le diagnostic porté en 2001 par Le Bourhis demeure largement pertinent : «D'autres politiques sectorielles ou territoriales [...], comme les politiques d'urbanisme des communes, les politiques industrielles et agricoles de l'État sont encore loin de prendre en compte systématiquement les interdépendances créées par l'eau. Une telle évolution impliquerait en effet une réorganisation du système politique et administratif local : modifications des zones de compétences et des allocations budgétaires, changement d'affectations de personnels, révision des routines bureaucratiques et des normes d'intervention. La régulation et le guidage administratif des activités agricoles, industrielles et d'urbanisme demeurent sous le contrôle de départements ministériels indépendants : pour ceux-ci, malgré des évolutions récentes et une réappropriation du discours environnemental, la question de l'eau est souvent pensée en opposition avec celle du développement économique et reste une priorité annexe» (Le Bourhis, 2001) ${ }^{24}$.

\section{Le régime institutionnel de l'eau : un régime intégré ?}

Tous ces éléments permettent finalement de qualifier le régime institutionnel de l'eau. Les deux critères permettant de caractériser ce régime sont, rappelons-le, la cohérence et l'étendue.

- La cohérence interne du système de droits de propriété (SDP) qui «fait référence au degré de clarté de la définition des droits de propriété et d'usage qui en résultent» (Varone et al., op. cit.) ${ }^{25}$ peut désormais être considérée comme élevée, dans la mesure où les ambigüités relevées sur le statut juridique des eaux souterraines, entre

24. Citation à la page 213.

25. Les références bibliographiques de ces auteurs sont inscrites dans la note 11 du présent article. Les spécificités des trois cohérences sont décrites à la page 10 de leur contribution « Régimes institutionnels de ressources et théorie de la régulation». le code civil et la loi sur l'eau de 1992, sont levées.

- La cohérence interne des politiques publiques (PP) qui «décrit surtout le degré de coordination entre les politiques d'exploitation et celles de protection des ressources naturelles » (Ibedem) demeure faible, eu égard notamment aux contradictions entre la politique de préservation de la qualité de l'eau et la politique agricole, qui demeure basée majoritairement sur un modèle de production intensive. La faiblesse des redevances payées par le secteur agricole comparée à celles des autres secteurs, réaffirmée en 2006 dans la LEMA, confirme ce point. La tendance générale est néanmoins à l'amélioration, si on observe les efforts entrepris depuis une quinzaine d'années pour lutter contre la pollution des eaux souterraines et leur surexploitation, mais elle demeure insuffisante pour répondre aux exigences communautaires, notamment en termes de qualité de l'eau.

- La cohérence externe « décrit la manière dont les deux composants d'un RIR sont articulés. Elle dépend en particulier de la correspondance entre le groupe cible d'une PP et le groupe de détenteurs de droits tels que définis dans la pratique par les SDP» (Ibedem). Si on ne prend en considération que les droits de propriété privés, la cohérence externe reste faible pour les mêmes raisons que celles évoquées précédemment. En effet, l'importance de la propriété privée, basée sur la propriété foncière, encore largement aux mains du secteur agricole en France, fait que les politiques publiques mises en œuvre, ciblées sur la conciliation des usages concurrents et la préservation de la ressource, ne prennent pas majoritairement pour groupe cible les agriculteurs. Cela se traduit, au niveau des mesures réglementaires, par une tendance des préfets à privilégier, lors des périodes de sécheresse, des restrictions d'usage pour les particuliers par rapport aux restrictions d'usage pour l'agriculture. Cependant, la mise en place de systèmes interannuels de quotas 
de prélèvements, basés sur le niveau piézométrique de la nappe de Beauce constitue un exemple illustrant la souplesse des mécanismes réglementaires négociés qui peuvent être mis en place (Petit, 2006).

$\mathrm{Au}$ niveau des redevances, les groupes cibles demeurent les usagers industriels et domestiques, même si là encore, l'application de redevances «prélèvements » et «pollution» pour le secteur agricole a été renforcée ces quinze dernières années. Enfin, les instruments participatifs montrent davantage de concordance et donc de cohérence entre les groupes cibles et les détenteurs de droits de propriété et d'usage. Cette tendance est renforcée par les limitations du droit de propriété privée introduites par les textes successifs, jusqu'à la reconnaissance du caractère domanial des eaux souterraines en 2006. Si on tient compte de l'évolution récente des droits de propriété, couplée à un renforcement des politiques publiques négociées (SAGE, contrat de nappe, etc.), la cohérence externe apparaît alors élevée.

Ainsi, grâce notamment aux évolutions notées ces dernières années, la cohérence du RIR a progressé et peut être qualifiée d'élevée. Toutefois, comme Calvo-Mendieta $(2005)^{26}$, le relève, «le niveau de cohérence $d u$ régime institutionnel de l'eau même s'il peut être qualifié d'élevé à la fin du millénaire, n'a pas encore atteint le maximum. »

Le deuxième critère, permettant de qualifier le régime institutionnel de l'eau est son étendue. Rappelons que " [1]'étendue d'un RIR fait référence au nombre de biens et services régulés par le régime à un temps donné » (Varone et al., 2008) ${ }^{27}$, c'est-à-dire à la prise en compte de l'ensemble des usages possibles de l'eau pour le domaine qui nous concerne. Comme le relève CalvoMendieta, l'étendue du régime institutionnel de l'eau a déjà atteint un maximum avant même l'adoption de la loi sur l'eau de 1992.

26. In Calvo-Mendieta, page 181.

27. In Varone et al., page 9.
La période récente ne fait que renforcer cette tendance, dans la mesure où l'eau est reconnue à la fois comme ressource et comme milieu. La loi sur l'eau de 1992, en posant le principe d'une gestion globale et équilibrée, en reconnaissant le caractère patrimonial de l'eau, en insistant sur la conciliation des usages, poursuit cette tendance qui sera aussi confirmée en 2006 dans la LEMA, portant à la fois sur l'eau et les milieux aquatiques.

Finalement, au regard des évolutions du régime juridique et des politiques publiques consacrées à la gestion des eaux souterraines, le RIR de l'eau peut être qualifié d'intégré. Les deux autres éléments d'analyse mobilisés (territoires de l'eau et dimension patrimoniale) soulignent néanmoins que cette intégration n'est pas totale et que la politique française de l'eau, souvent érigée en modèle à l'échelle internationale, comme pionnière en matière de gestion intégrée, ne remplit qu'imparfaitement tous les défis posés par cette notion.

\section{Conclusion}

En proposant d'analyser, au regard de la GIRE, la politique française de gestion des eaux souterraines depuis le début des années 1990 , on pourrait croire que cette notion constitue à nos yeux un objectif en soi. Or, il n'en est rien. Si la notion de GIRE nous intéresse, c'est parce qu'elle participe d'un mouvement, à l'échelle internationale, qui revendique la prise en compte du développement durable, du patrimoine, de nouvelles formes de gouvernance, autant de concepts « valises » qui ouvrent le champ des problématiques scientifiques, notamment lorsqu'on les confronte aux mécanismes de décision mis en œuvre. En analysant l'évolution des droits de propriété et des politiques publiques appliquées à un domaine particulier - celui des eaux souterraines - et en relevant une inflexion notable vers davantage d'intégration, on ne parcourt qu'une partie du chemin. Nous pouvons soutenir, 
comme le fait Theys $(2000)^{28}$ à propos du développement durable, que la GIRE désigne un "principe normatif sans normes » et qu'elle ne peut donc être $a$ priori définie scientifiquement. Ceci explique pourquoi cette notion cristallise autant de débats et implique des négociations, un renouvellement des procédures de

28. In Theys, page 22. prise de décision et finalement de l'action publique. Toutes ces mutations justifient qu'à côté d'un travail sur le(s) sens de la GIRE, les transformations institutionnelles et politiques à l'œuvre, à toutes les échelles, soient analysées. Notre contribution tente d'apporter quelques clés pour comprendre ces mutations, mais nous sommes convaincus que seul un travail interdisciplinaire et collectif permettra d'avancer dans cette compréhension.

\section{RÉFÉRENCES BIBLIOGRAPHIQUES}

Babin D., Antona M., Bertrand A., Weber J. (2002). Gérer à plusieurs des ressources renouvelables. Subsidiarité et médiation patrimoniale par récurrence. In CormierSalem M.-C., Juhé-Beaulaton D., Boutrais J., Roussel B. (Dir.), « Patrimonialiser la nature tropicale. Dynamiques locales, enjeux internationaux ", Paris, IRD Éditions, collection : Colloques et séminaires, p. 79-99.

Barraqué B. (1997). Les agences de l'eau et la question du patrimoine commun en France et en Europe. Paris, rapport pour le Commissariat Général du Plan.

Barraqué B., (1999). Le Ministère de l'Environnement et les Agences de l'eau. In Lascoumes P. (Dir.), «Instituer l'environnement. Vingt-cinq ans d'administration de l'environnement », Paris, L'Harmattan, collection : Logiques politiques, p. 103-127.

Barrère C., Barthélemy D., Nieddu M., Vivien F.-D. (2005). Réinventer le patrimoine. De la culture à l'économie, une nouvelle pensée du patrimoine? Paris, L'Harmattan, collection : Gestion de la culture et du secteur non lucratif.

Barthélemy D., Nieddu M., Vivien F.-D. (2005). Économie patrimoniale, identité et marché. In Barrère C., Barthélemy D., Nieddu M., Vivien F.-D. (Dir.), «Réinventer le patrimoine. De la culture à l'économie, une nouvelle pensée du patrimoine? ", Paris, L'Harmattan, collection : Gestion de la culture et du secteur non lucratif, p. 121-150.

Billet P. (2001). La régulation juridique des conflits liés à la gestion des eaux souterraines. Revue juridique de l'Environnement, $\mathrm{n}^{\circ}$ 3, p. 401-416.

Biswas A.-K. (2004). Integrated Water Resources Management: a Reassessment. Water International, vol. 29, $\mathrm{n}^{\circ} 2$, p. 248-256.

Busca D., Gendre C., Salles D., Zelem M.-C. (2003). Comment intégrer les contraintes environnementales en agriculture? In Gendron C., Vaillancourt J.-G. (Dir.), «Développement durable et participation publique. De la contestation écologiste aux défis de la gouvernance », Montréal, Les Presses de l'Université de Montréal, p. 307-324.

Calvo-Mendieta I. (2005). L'économie des ressources en eau : de l'internalisation des externalités à la gestion intégrée. L'exemple du bassin-versant de l'Audomarois. Lille, Université de Sciences et Technologies, thèse en Sciences Économiques, décembre, $345 \mathrm{p}$.

Comte J.-P., Retkowski Y., Sallenave M. (1995). Les SDAGE, les SAGE et l'eau souterraine. Conseil général des Mines, groupe de travail : Schémas d'aménagements SDAGE et SAGE, rapport final, décembre. 
Ghiotti S. (2007). Les territoires de l'eau. Gestion et développement en France. Paris, CNRS Éditions, collection : Espaces et milieux.

Institut français de l'environnement (IFEN) (2004). L'état des eaux souterraines en France. Aspects quantitatifs et qualitatifs. Orléans, IFEN, collection : Études et travaux, $\mathrm{n}^{\circ} 43,37 \mathrm{p}$.

Le Bourhis J.-P. (2001). L'eau, terrain d'expérimentation. In «L'environnement, question sociale. Dix ans de recherches pour le ministère de l'Environnement ", Paris, Éditions Odile Jacob, p. 207-214.

Llamas R.-M. (1998). La protection des eaux souterraines en Espagne. In Barraqué B., Theys J. (Dir.), «Les politiques d'environnement. Évaluation de la première génération », Paris, Éditions Recherches, p. 119-136.

Martin Y. (1996). Rapport sur la gestion durable des eaux souterraines. Conseil général du GREF, 29 janvier, 30 p.

Mermet L. (2007). Projets, tentations et fantasmes hathrôlogiques : le syndrome patrimonial. Communication dans le cadre du cycle RES - Recherche Environnementale sur la Société, Séance 10, Montpellier, Engref, 2 février, http://www.rgte.centre-cired.fr/reel-rgte/

Montgolfier (de) J., Natali J.-M. (1987). Le patrimoine du futur. Approches pour une gestion patrimoniale des ressources naturelles. Paris, Economica, collection : économie agricole et agroalimentaire.

Narcy J.-B. (2004). Pour une gestion spatiale de l'eau. Comment sortir du tuyau? Bruxelles, P.I.E. Peter Lang, collection : EcoPolis, $n^{\circ} 4$.

Ollagnon H. (1979). Propositions pour une gestion patrimoniale des eaux souterraines : l'expérience de la nappe phréatique d'Alsace. Paris, la documentation française, Bulletin interministériel pour la rationalisation des choix budgétaires, $\mathrm{n}^{\circ} 36$, p. 33-73.
Ollagnon H. (1987). À la rencontre des approches théoriques et pragmatiques de la gestion de la nature : l'audit patrimonial de type «systèmes-acteurs ». Cahier du Germes, L'environnement dans l'analyse et la négociation des projets, p. 495-511.

Petit O., Baron C. (2009). Integrated Water Resources Management: From General Principles to its Implementation by the State. The case of Burkina Faso. Natural Resources Forum, Vol. 33, ${ }^{\circ} 1$, forthcoming.

Petit O. (2006). La gouvernance de la nappe de Beauce. Du conflit d'usage à la coopération. Actes du colloque international GECOREV «Gestion concertée des ressources naturelles et de l'environnement ", Guyancourt, université de Versailles Saint-Quentin-en-Yvelines.

Petit O. (2009). Eau et développement durable : vers une gestion intégrée ? In Grumiaux F., Matagne P. (Dir.), "Le développement durable sous le regard des sciences et de l'histoire : de la réflexion aux pratiques éducatives et de formation », Paris, L'Harmattan.

Salles D. (2006). Les défis de l'environnement. Démocratie et efficacité. Paris, Syllepse, collection : Écologie et Politique.

Salles D., Zelem M.-C. (1997). Processus de négociation des contrats de rivière dans le bassin Adour-Garonne. Université Toulouse Le Mirail, Maison de la Recherche, CERTOP-CNRS / GIS ECOBAG.

Theys J. (2000). Un nouveau principe d'action pour l'aménagement du territoire? Le développement durable et la confusion des (bons) sentiments. In Theys J. (Coord.), «Développement durable ville et territoires. Du concept à sa mesure et à sa mise en auvre : innover et décloisonner pour anticiper les ruptures », Notes du Centre de Prospective et de Veille Scientifique, $n^{\circ} 13$, DRAST, ministère de l'Équipement, p. 8-33. 